\title{
Optimisation and harmonisation: two sides of the same coin?
}

\author{
Ronald Boellaard
}

Received: 18 April 2013 /Accepted: 19 April 2013 /Published online: 15 May 2013

(C) Springer-Verlag Berlin Heidelberg 2013

${ }^{18}$ F-Fluorodeoxyglucose (FDG) positron emission tomography/computed tomography (PET/CT) examinations are executed for several oncological applications [1]. In most cases PET/CT studies are performed with a diagnostic intent, often interpreted using visual inspection of the images. The primary task of the observer is e.g. to identify unknown primary tumours, lymph node involvement and the assessment of distant metastasis. It has been shown that FDG PET/CT is an important tool for either up or down staging of lung cancer patients with a direct impact on patient management [2]. The uptake of FDG can be used as a prognostic factor both in the clinical setting and in trials [3]. In addition to diagnosis and staging, FDG PET/CT is used to measure treatment response. Here residual FDG uptake or changes in uptake during or after treatment are used as predictive factors [4-6]. To this end, criteria to measure FDG uptake changes and/or metabolic response have been proposed [7, 8]. Finally, PET/CT studies performed at follow-up are used to assess presence or absence of recurrence of disease. Apart from the various applications of FDG PET/CT listed above, there are many other utilities of FDG PET/CT. For example, it can be used for radiation oncology purposes [9-12], e.g. by identifying areas with high metabolic activity that may require a boost of radiation to improve treatment outcome.

In clinical practice most FDG PET/CT studies are interpreted by visual inspections. Particularly when these studies are conducted for diagnostic purposes the main objective of the reader is to identify the absence or presence of malignant lesions or residual disease [1]. Yet, there is a strong interest to use FDG PET/CT in a quantitative manner. The uptake of FDG, expressed by standardised uptake values (SUV), has been shown to be useful prognostic and predictive measures [6]. Moreover, changes in SUV can be used to

\section{R. Boellaard $(\bowtie)$}

Department of Radiology \& Nuclear Medicine, VU University

Medical Centre, De Boelelaan 1117, 1081 HV Amsterdam,

The Netherlands

e-mail: r.boellaard@vumc.nl measure treatment efficacy, already early within a few weeks after start of treatment and may then impact subsequent patient management $[5,13]$.

In drug trials quantitative PET/CT will play an important role at various stages during the drug development procedure. At baseline FDG PET/CT is used to identify subjects eligible for the treatment and quantitative uptake measures can be used for stratification and/or to identify target lesions. During treatment changes in SUV of these target lesions can then be used to assess drug efficacy. FDG PET/CT has therefore the potential to become an important imaging biomarker in trials [13]. However, there are several criteria that must be met in order to use and approve any imaging modality as an imaging biomarker, i.e. before it can be used and is accepted as a surrogate end point in trials. Some of these important criteria are repeatability (in one patient using the same PET/CT system) and reproducibility (between patients, systems and institutions) of FDG PET/CT performance, analysis and interpretation. Along with these requirements, standardisation of acquisition and data analysis procedures is warranted to be creditable for referring physicians [14] and is demanded by authorities (such as the European Medicines Agency and the US Food and Drug Administration) before they can be approved as imaging biomarkers [15].

From the above given overview it is clear that there may be two different sets of requirements for the quality and quantitative accuracy and precision of FDG PET/CT studies. Optimisation relates to the task at hand, which in turn relates to a figure of merit to be optimised. On the one hand there is a need to generate $\mathrm{PET} / \mathrm{CT}$ data with image characteristics that are optimal for visual interpretation, i.e. the task. The figure of merit to be optimised is then 'detectability of a malignant lesion'. The latter is a combined measure of sensitivity and specificity. It is plausible that by improving spatial resolution and reducing image noise, thereby improving contrast to noise ratio, the diagnostic quality of FDG PET/CT may improve $[16,17]$, provided that $\mathrm{PET} / \mathrm{CT}$ readers have become familiar with the new characteristics of the PET/CT images resulting from improved technologies. On the other hand when $\mathrm{PET} / \mathrm{CT}$ is to be used in a quantitative manner in a multicentre 
setting a different task is required. The task at hand is to derive quantitative measures, such as SUV, from the PET/CT studies in a harmonised manner. The figure of merit to be optimised relates to the accuracy and precision of SUVs across multiple institutions having different PET/CT systems [18-20]. Harmonisation in this case means that sites are able to generate SUVs with a comparable and known accuracy and precision in a manner that is feasible for all sites in a multicentre setting. The latter aim was one of the main objectives of the European Association of Nuclear Medicine guideline for quantitative FDG PET/CT imaging (EANM FDG PET/CT guideline) [18]. This philosophy also formed the basis for defining a quality control programme with harmonising performance criteria, which now runs across Europe by the EARL FDG PET/CT accreditation programme (http:// earl.eanm.org/cms/website.php).

The process of optimisation for different tasks does not necessarily converge to the same solution. In fact, with the introduction of new PET technologies, such as time-of-flight and resolution modelling during reconstruction or point spread function reconstruction, PET/CT data with higher resolution and better contrast to noise characteristics can be acquired. Often, use of these new technologies is preferred by the nuclear medicine physicians for visual assessment of the images [16, 17]. At the same time use of these new technologies may result in a wider variability (and change) of SUV across different centres [16, 19, 21]. Moreover, use of the maximum SUV ( $\left.\mathrm{SUV}_{\max }\right)$, being the de facto standard in many studies, in combination with these new technologies may even worsen accuracy and precision [19, 22].

Are image quality optimisations for different tasks two sides of the same coin? In other words do we have to choose between one and the other? Or can we acquire data in such a way that we can benefit from new technologies to enhance visual assessments and at the same time generate quantitative measures that are robust and reliable in a multicentre setting? This important issue is addressed in the paper by Lasnon et al. [22] in this issue of the European Journal of Nuclear Medicine and Molecular Imaging.

Lasnon et al. [22] show that by performing two reconstructions from a single PET/CT acquisition it is possible to generate two PET data sets, one optimal for visual inspection and another meeting the multicentre requirements of the EANM FDG PET/CT guideline. In fact, the authors show an excellent agreement between quantitative results for data generated without use of new technologies and those reconstructed with new technologies and including additional processing during reconstruction. This paper presents an important message, namely that we can benefit from new technologies without compromising quantitative robustness as a prerequisite for using FDG PET/CT as an imaging biomarker.

Lasnon et al. [22] performed two reconstructions to generate two separate data sets. Reconstructions today are still time consuming and often only a single reconstruction can be done during the overall time of the PET/CT acquisition. As physicians need to be able to quickly inspect PET/CT images after concluding the PET acquisition, a strategy of performing two reconstructions could potentially cause problems with workflow in a busy clinical environment. There are, however, several solutions to bypass workflow issues. One is to do the additional reconstructions offline overnight meaning that quantitative reads will be delayed by 1 day. Again this solution is not practical in routine clinical practice. However, Lasnon et al. [22] demonstrated that the only necessary step consisted of post-processing the PET images using a Gaussian filter of $7 \mathrm{~mm}$ full-width at halfmaximum (FWHM). This very simple post-processing step would, by itself, not take longer than a few seconds. The lack of functionality of generating a second data set by simple post-processing on their PET/CT system or display station has probably forced the authors into doing a second time-consuming reconstruction process. Therefore, it would be extremely useful if PET/CT vendors were able to implement additional post-processing functionality as part of their standard reconstruction process [19], which would create an additional post-processed image data set. Preferably, the post-processing functionality could be automatically attached to acquisition and reconstruction protocols upon user request, after which this second data set is automatically generated following a singe image acquisition and reconstruction. The applied filter settings, along with the original reconstruction parameters, should preferably be stored in the Digital Imaging and Communications in Medicine (DICOM) header. The latter allows analysis software, observers and trial coordinators to verify that (secondary) data sets comply with required harmonised image characteristics.

Lasnon et al. [22] performed their evaluations using $\mathrm{SUV}_{\max }$ and the SUV based on a $50 \%$ isocontour volume of interest $\left(\mathrm{SUV}_{\text {mean }}\right)$. It is clear that use of $\mathrm{SUV}_{\text {mean }}$ provides $\mathrm{SUV}$ recoveries closer to one, i.e. more accurate SUVs, on average when applied to PET images based on new reconstruction technologies. Yet, the relation between SUV recovery and sphere size still shows the same amount of variability as those obtained according to the EANM FDG PET/CT guideline (Supplementary Fig. 1 in [22]). Although use of SUV mean could be attractive, there is no (global) consensus on which volume of interest strategy to use. There are many different volume of interest methods being explored and there is a wide variability in metabolic volume and SUV performance as functions of image characteristics and volume of interest method applied [23]. Moreover, different implementations of supposedly the same method exist across different display and analysis workstations. Finally, most widely available volume of interest methods are only suitable in cases of near uniform tracer uptake. Consequently, $\mathrm{SUV}_{\max }$ has become the de facto standard for quantitative assessment of tracer uptake. Despite the 
reported upward bias in $\mathrm{SUV}_{\max }[24,25]$, which becomes even more pronounced with new PET technologies (Supplementary Fig. 1 in [22]), it has some attractive features. Conceptually it may represent the most metabolically active part of the tumour, which might contain the most prognostic or predictive value. Zander et al. [6] showed that use of $\mathrm{SUV}_{\max }$ is a suitable predictive parameter and that it performed equally well compared to other SUVs. Moreover $\mathrm{SUV}_{\max }$ is uniquely defined, i.e. there is only one $\mathrm{SUV}_{\max }$ in each lesion. It is, in principle, observer independent provided a reasonable (manual) delineation of the tumour was made. Moreover, most display stations are able to generate $\mathrm{SUV}_{\max }$ data. Consequently, $\mathrm{SUV}_{\max }$ is the most widely used, primarily because of ease of use and feasibility of generating these data in multicentre studies.

An alternative to $\mathrm{SUV}_{\text {max }}$ might be the use of the so-called peak SUV $\left(\mathrm{SUV}_{\text {peak }}\right)$ [7]. $\mathrm{SUV}_{\text {peak }}$ is based on a spherical volume of interest having a volume of $\sim 1 \mathrm{ml}$, positioned over the lesion such to obtain the highest average value. By averaging over $1 \mathrm{ml}$ volume the upward bias as a function of image noise seen with $\mathrm{SUV}_{\max }$ may be avoided [25]. At the same time $\mathrm{SUV}_{\text {peak }}$ may be less sensitive to underlying image characteristics as the dimensions of the peak volume of interest act as a smoothing filter [25]. Use of $\mathrm{SUV}_{\max }$ and $\mathrm{SUV}_{\text {peak }}$ in combination with the strategy described by Lasnon et al. [22] should therefore become the standard methodological approach in quantitative multicentre FDG PET/CT studies. Key aspects are [18] harmonisation of imaging procedures (patient preparation etc.), harmonisation of data analysis methods $\left(\mathrm{SUV}_{\max }\right.$ and $\left.\mathrm{SUV}_{\text {peak }}\right)$ and harmonisation of image characteristics that coexist with those needed for optimal diagnostic performance. Lasnon et al. [22] have shown that the latter can be achieved in a very simple manner.

\section{References}

1. Fletcher JW, Djulbegovic B, Soares HP, Siegel BA, Lowe VJ, Lyman GH, et al. Recommendations on the use of $18 \mathrm{~F}-\mathrm{FDG}$ PET in oncology. J Nucl Med 2008;49(3):480-508.

2. Czernin J, len-Auerbach M, Schelbert HR. Improvements in cancer staging with PET/CT: literature-based evidence as of September 2006. J Nucl Med 2007;48 Suppl 1:78S-88.

3. Geus-Oei LF, van der Heijden HF, Corstens FH, Oyen WJ. Predictive and prognostic value of FDG-PET in nonsmall-cell lung cancer: a systematic review. Cancer 2007;110(8):1654-64.

4. Kobe C, Scheffler M, Holstein A, Zander T, Nogoya L, Lammertsma AA, et al. Predictive value of early and late residual $18 \mathrm{~F}$-fluorodeoxyglucose and $18 \mathrm{~F}$-fluorothymidine uptake using different SUV measurements in patients with non-small-cell lung cancer treated with erlotinib. Eur J Nucl Med Mol Imaging 2012;39(7):1117-27.

5. Weber WA. PET for response assessment in oncology: radiotherapy and chemotherapy. Br J Radiol 2005;Suppl 28:42-9.

6. Zander T, Scheffler M, Nogova L, Kobe C, Engel-Riedel W, Hellmich M, et al. Early prediction of nonprogression in advanced non-small-cell lung cancer treated with erlotinib by using [(18)F] fluorodeoxyglucose and [(18)F]fluorothymidine positron emission tomography. J Clin Oncol 2011;29(13):1701-8.

7. Wahl RL, Jacene H, Kasamon Y, Lodge MA. From RECIST to PERCIST: evolving considerations for PET response criteria in solid tumors. J Nucl Med 2009;50 Suppl 1:122S-50.

8. Young H, Baum R, Cremerius U, Herholz K, Hoekstra O, Lammertsma AA, et al. Measurement of clinical and subclinical tumour response using [18F]-fluorodeoxyglucose and positron emission tomography: review and 1999 EORTC recommendations. European Organization for Research and Treatment of Cancer (EORTC) PET Study Group. Eur J Cancer 1999;35(13):1773-82.

9. Chiti A, Kirienko M, Grégoire V. Clinical use of PET-CT data for radiotherapy planning: what are we looking for? Radiother Oncol 2010;96(3):277-9.

10. Gregoire V, Chiti A. PET in radiotherapy planning: particularly exquisite test or pending and experimental tool? Radiother Oncol 2010;96(3):275-6.

11. Roels S, Slagmolen P, Nuyts J, Lee JA, Loeckcx D, Maes F, et al. Biological image-guided radiotherapy in rectal cancer: challenges and pitfalls. Int J Radiat Oncol Biol Phys 2009;75:782-90.

12. Thorwarth D, Schaefer A. Functional target volume delineation for radiation therapy on the basis of positron emission tomography and the correlation with histopathology. Q J Nucl Med Mol Imaging 2010;54(5):490-9.

13. Weber WA. Chaperoning drug development with PET. J Nucl Med 2006;47(5):735-7.

14. Karantanis D, Kalkanis D, Allen-Auerbach M, Bogsrud TV, Subramaniam RM, Danielson A, et al. Oncologic 18F-FDG PET/CT: referring physicians' point of view. J Nucl Med 2012;53(10):1499-505.

15. Weber WA. Positron emission tomography as an imaging biomarker. J Clin Oncol 2006;24(20):3282-92.

16. Andersen FL, Klausen TL, Loft A, Beyer T, Holm S. Clinical evaluation of PET image reconstruction using a spatial resolution model. Eur J Radiol 2013;82(5):862-9.

17. Lasnon C, Hicks RJ, Beauregard JM, Milner A, Paciencia M, Guizard $\mathrm{AV}$, et al. Impact of point spread function reconstruction on thoracic lymph node staging with $18 \mathrm{~F}-\mathrm{FDG}$ PET/CT in nonsmall cell lung cancer. Clin Nucl Med 2012;37(10):971-6.

18. Boellaard R, O'Doherty MJ, Weber WA, Mottaghy FM, Lonsdale MN, Stroobants SG, et al. FDG PET and PET/CT: EANM procedure guidelines for tumour PET imaging: version 1.0. Eur J Nucl Med Mol Imaging 2010;37(1):181-200.

19. Boellaard R. Need for standardization of $18 \mathrm{~F}-\mathrm{FDG}$ PET/CT for treatment response assessments. J Nucl Med 2011;52 Suppl 2:93S-100.

20. Boellaard R. Standards for PET image acquisition and quantitative data analysis. J Nucl Med 2009;50 Suppl 1:11S-20.

21. Beyer T, Czernin J, Freudenberg LS. Variations in clinical PET/CT operations: results of an international survey of active PET/CT users. J Nucl Med 2011;52:303-10.

22. Lasnon C, Desmonts C, Quak E, Gervais R, Do P, Dubos-Arvis C, et al. Harmonizing SUVs in multicentre trials when using different generation PET systems: prospective validation in non-small cell lung cancer patients. Eur J Nucl Med Mol Imaging 2013. doi:10.1007/s00259-013-2391-1.

23. Cheebsumon $\mathrm{P}$, Yaqub $\mathrm{M}$, van Velden FH, Hoekstra OS, Lammertsma AA, Boellaard R. Impact of [(18)F]FDG PET imaging parameters on automatic tumour delineation: need for improved tumour delineation methodology. Eur J Nucl Med Mol Imaging 2011;38(12):2136-44.

24. Boellaard R, Krak NC, Hoekstra OS, Lammertsma AA. Effects of noise, image resolution, and ROI definition on the accuracy of standard uptake values: a simulation study. J Nucl Med 2004;45(9):1519-27.

25. Lodge MA, Chaudhry MA, Wahl RL. Noise considerations for PET quantification using maximum and peak standardized uptake value. J Nucl Med 2012;53(7):1041-7. 